\title{
The Role of Dentistry in Disaster Management and Victim Identification: An Overview of Challenges in Indo-Nepal Scenario
}

\author{
Shubha Ranjan Dutta ${ }^{1} \cdot$ Purnima Singh $^{2} \cdot$ Deepak Passi $^{3} \cdot$ Don Varghese ${ }^{4}$. \\ Sarang Sharma ${ }^{5}$
}

Received: 14 June 2015/Accepted: 21 March 2016/Published online: 21 April 2016

(C) The Association of Oral and Maxillofacial Surgeons of India 2016

\begin{abstract}
Background Recently, natural disasters and terrorist activities have been leading to mass casualty situations unexpectedly around the globe. In addition to the traditional emergency medical services centering around medically trained and paramedic personnel, dental practitioners having vital skills and attributes may be important in responding to a mass casualty situation.

Objective This paper aims at discussing the role of dentists in disaster management and the role of forensic odontology in the disaster victim identification (DVI), its status in India and some suggestions to develop the plans for same.

Materials and methods Articles were searched in various medical databases such as Google Scholar, Pubmed Central, Sciencedirect,Wiley online Library, Scopus, Copernicus to gather all relevant information on the subject. Various keywords were used as search tool such as 'Mass disaster', 'Forensic odontology', 'Victim identification'.
\end{abstract}

Shubha Ranjan Dutta

drshubharanjand@gmail.com

1 Department of Oral and Maxillofacial Surgery, M B Kedia Dental College, Tribhuwan University, Chhapkaiya, Birgunj-2, Nepal

2 Department of Physiology, M B Kedia Dental College, Tribhuwan University, Chhapkaiya, Birgunj, Nepal

3 Department of Oral and Maxillofacial Surgery, E. S. I. C. Dental College and Hospital, Rohini, Delhi, India

4 Department of Oral and Maxillofacial Surgery, Jodhpur Dental College and Hospital, Jodhpur, Rajasthan, India

5 Department of Conservative Dentistry and Endodontics, E. S. I. C. Dental College and Hospital, Rohini, Delhi, India
Results The search resulted in total of 170 articles which we reviewed. Due to limitation to the list of references we have constricted our review to only 39 articles for more informative literature and supported the topic of the present manuscript 'The Role of Dentistry in Disaster Management and Victim Identification: An Overview of Challenges in Indo-Nepal Scenario' more specifically.

Conclusion Every disaster is unique and involves interplay of different factors and circumstances such as nature of disaster, number of victims and extent of body fragmentation that ultimately challenges the disaster response planning. Apart from the victim recovery and evacuation, the disaster response planning must include the established procedures for the identification of the victims of the disaster. The identification of victims essentially relies on forensic anthropology, radiology, DNA typing and fingerprints, as well as odontology.

Keywords Mass disaster - Forensic odontology · Victim identification

\section{Introduction}

Terrorist attacks and other catastrophic events create demands that far exceed the capacity of the medical and public healthcare systems to respond. The increased number of casualties decreases the ability to respond, which is further delayed by fear and the urgent need of information. All disasters independent of its origin reflect unique environments, needs and risks within a particular state, jurisdiction and community [1]. Over the past couple of years, the governments of countries like India and Nepal have brought a paradigm shift in approach to disaster management. The approach has been translated into a national 
disaster framework and has been shared with all sub-divisional governments and administrations having a common scheme. The roles of traditional first responders shift in case of severe disaster events, particularly when the number of victims overflows the hospitals and clinics. In such situations, dentists and dental auxiliaries play a vital role in response to disaster by wide range of skill sets based on personal experience, training and enthusiasm [2]. Dentists are one of the important constituents of health team, yet their role or utility has not been emphasized lately. Oral healthcare personnel including dentists can be utilized successfully for the emergency medical response system in building the required partnerships, to identify and garner resources, and in facilitating training, development of suitable policy, surveillance and evaluation [3].

\section{Dental Professionals and Response}

In India and abroad, dentists are subjected to medical and surgical training during their pre-doctoral education that makes them useful in disaster management situations. They are also trained for handling medical emergencies in dental clinics and hospitals such as performing minor surgery, dispensing of drugs, giving injections and administration of anaesthesia [2]. Specialized dental surgeons or oral and maxillofacial surgeons (OMFS) are also capable of administering anaesthetic and surgical services to patients with many types of injuries. OMFS apart from treating dentoalveolar injuries and facial fractures are also efficient in handling trauma patients and can provide first aid, including endotracheal intubation and cardiopulmonary resuscitation [4].

The initial training obtained by the dental professionals during their graduate degree makes them important in terms of catastrophic events in the following ways [5]:

- Well trained in sterilization techniques and infection control methods

- Trained in drug delivery methods including injection

- Expert in suturing techniques and bleeding controlling methods

- Proficient in participating in interdisciplinary professional groups, and

- Trained in handling uncomfortable patients

However, these services can also be provided by other health professionals like the physicians, pharmacists and nursing professionals. But in a mass disaster scenario, when there is shortage of physicians the role of dentist becomes more important as only dentists have the standard training for carrying out these services both at undergraduate and at postgraduate level and can be a best substitute. No other profession has undergraduate training that so closely parallels that of the physician as does the training of the dentist.

By virtue of training and practical experience, dentists possess a high degree of manual dexterity together with excellent hand and eye coordination in the treatment of patients, frequently under extreme conditions of pain and infection.

Moreover, the primary role played by dentists in disasters has been in the form of forensic odontology $[6,7]$ and the assistance of dental members in Disaster Mortuary Operational Response Teams [7]. Dentists are also employed in the armed forces sector for their valuable services [8]. Instead, an additional training is required in medical and nursing education for performing these functions [9]. Thus, to incorporate the participation of other non-physician and nurse healthcare groups such as dental hygienists, pharmacists, podiatrists, and mental health workers in such services, particular areas of surge manpower need to be identified and the underlying training required by healthcare professionals including dentists; thus, surge roles can be addressed and the necessary skill sets can be incorporated into the education of these professionals.

The services rendered by dental professionals during a major public health disaster are described in detail as follows:

\section{Dental Surveillance}

Bioterrorism attacks and pandemics often have relatively indistinguishable beginnings and ends and unpredictable effects on a population. Surveillance is important in conditions when there is increased influx of patients with symptoms of paralysis or neurology, or infection, which is non-traumatic. The presence of dental offices in nearly every community makes it very important in a catastrophic disaster. Thus, dentists can be a part of effective surveillance network by getting updated on the information on unusual syndromes in the community as well as unusual clinical presentations [10]. For example, dentists can notify the public health authorities about the presence of patients with intra-oral or cutaneous lesions or both, thus facilitating the early detection of a bioterrorism attack or spread of a pandemic infection. In this way, an early detection of an infectious agent in a population can lead to reduction in mass casualties by prompt prevention and therapeutic methods. This criterion also makes dentists unique amongst all other health professionals.

\section{Dental Offices Acting as Medical Sites}

Dental offices are equipped with potentially useful equipment and supplies that prepare it to serve as decentralized 
auxiliary hospitals in case the need arises [6]. Availability of air and suction lines, X-ray equipment and sterilizing techniques in dental offices can be used as self-contained alternate medical sites if hospitals are under attack or are unsafe because of widespread infection associated with biological weapons [10]. No other health professional has such well-equipped clinic to provide as alternative medical site for use during an event of catastrophic disaster.

\section{Distribution of Medication}

During medical emergency situations, it may be possible that physicians, nurses and pharmacists are not able to effectively prescribe or dispense the medication necessary in the critical and appropriate time. This calls for a dentist who can prescribe and dispense the required medications, after the outbreak of the disease has been determined by the physician and public health officials managing it. Patients can be monitored by dentists for adverse reaction and side effects, and if necessary, they are also referred to physicians for treatment of untoward effects from the medications $[6,7]$. Dentists can also be a source of information about the medication and its proper use, side effects and the need for compliance for the patient [11].

\section{Immunization}

In major metropolitan areas, where the spread of communicable disease is facilitated, this effort may involve millions of people. The number of physicians and nurses available may not be able to implement immunization program in limited time. In urgent programs, trained dentists can participate in mass immunization programs. Dental clinics can also be considered as immunization sites to minimize the concentration of potentially infected patients [7, 12].

\section{Definitive Treatment}

Dentists have training and experience in many areas that may be a part of casualty care in mass casualty events. As mentioned earlier, OMFS are appropriately trained to provide first aid, endotracheal intubation, cardiopulmonary resuscitation as well as anaesthetic and surgical services to patients with different injuries [4]. Dentists can identify the infectious disease that causes mass casualties and can recognize the sign and symptoms of that disease which may help to identify the afflicted patients [8]. Dentists regularly collect salivary samples, nasal swabs, or other specimens for laboratory processing, leading to proper diagnosis, information about progress of the treatment and the status of the infection of patient [13]. Thus, it is suggested that civilian dentists should be trained along with dental personnel in armed services to form a good team with their medical counterparts and augment the emergency care to large number of causalities that may be generated in severe disaster including major earthquake $[9$, 14].

\section{Triage Services}

Triage is derived from French word 'trier', which means to sort out. It is applied to various situations ranging from everyday triage of emergency department patients to largescale disasters [12]. During a mass casualty event when there may be deficiency of personnel, facilities, and medical supplies, it may not be possible to give immediate treatment for all casualties. In such situations, triage must be done to prioritize treatment plan. Dentists can provide assistance in these treatment procedures without further training. This provides additional help to physicians providing definitive care for patients most urgently in need rather than screening casualties. Dental offices could serve as triage centres if needed [15].

Triage consists of rapid classification of the injured on the basis of the severity of their injuries and the likelihood of their survival [3].

Red colour indicates high priority treatment or transfer. Yellow colour signals medium priority.

Green colour indicates ambulatory patients.

Black colour is for dead or moribund patients.

\section{Forensic Assistance}

Forensic odontology continues to be a crucial element in nearly all mass disasters irrespective of its incidence, viz. natural or accidental. This is one area which makes the dental professionals unique and far more important than others, especially in catastrophic events. The multiple functions of this unique discipline can range from the identification of human remains to mass disaster management, including the assessment of bite marks and patterned skin injuries along with the use of dental materials in the examination of evidence [16]. Dental identifications have always played a key role in victim identification during natural and manmade disaster events, particularly mass casualty events normally associated with aviation disasters [17]. A dental record is a well-structured document recording the details related to the history of the illness, physical examination, diagnosis, treatment, and management of a patient. The legislation compels dental professionals to produce and maintain adequate patient records. Dental records can also find their use in forensic cases, teaching and research, as well as in legal matters. Comprehensive and accurate records are a vital part of dental 
practice. However, primarily dental records are maintained to deliver quality treatment and care to patients and for follow-up.

\section{Supporting Other Health Professionals}

It is the responsibility of the private practitioners and local dental societies to make the local emergency response planners aware of the services rendered by the dental profession at the time of disaster [8]. As already discussed, in response to a declared medical emergency, the role of medical professionals can be augmented by dentists and their dental auxiliaries. Dentists can be recruited to provide certain services that only physicians can do when there is inadequate local medical resources to cope adequately with increasing number of patients. Dentists can enhance the surge capacity of the local medical system until another team of physicians can arrive or the demand for immediate care decreases [2]. Nurses or other healthcare professionals lack such training as required for disaster response.

\section{Infection Control}

Dentists and dental auxiliaries practice appropriate methods of infection control in their clinic on a daily basis. They are well versed and well practiced in infection control methods and can bring their expertise to mass casualty situations, particularly related to control of infectious agents, thereby limiting the spread of infection through patients and responders who are rendering assistance. Dentists can help to prevent decontamination casualties from certain bioterrorism attacks in which contact with patients' clothing or skin surfaces may also lead to the spread of infection to caregivers. Dentists already familiarized with the disaster mortuary activities can be useful in managing the remains of victims whose death is a result of the event, particularly infectious events [18].

\section{Quarantine}

During a pandemic or after bioterrorism attack with a communicable agent, the spread of the disease can be controlled or prevented by imposing strict quarantine restrictions on the geographic area contaminated and its environment. The duration of quarantine depends upon the incubation time of the agent and other factors. Before the existence of the quarantine area, wide contamination is already established which can infect the primary care providers directly or through contact with patients under care. This could lead to disability or even death. Dentists may not be similarly infected by patients because dental appointments are not given to infected patients. Dentist may also provide primary health care for people in the quarantined area [19].

In Japan, dentists were employed for the examination of the teeth of thousands of bodies who died in the March 11, 2011 earthquake and tsunami in an effort to help identify them. Immediately after the great disaster the JDA's Earthquake Disaster Response Headquarters was set up by the Japan Dental Association (JDA). Under the presidentship of Dr. Mitsuo Okubo, JDA performed various activities like dispatch of dentist teams to the affected areas for identification of disaster victims, keeping a check on the safety of JDA members and their families, survey of the status of dental offices as well as hospitals and related oral health facilities, making plans to send mobile clinics to deliver oral health care at shelters and making the shipment (using trucks arranged by the government) of oral health care products, hand instruments, devices and dental materials to be delivered to the dentists already under progress $[20,21]$.

\section{Disaster Victim Identification and Its Relation with Dentistry}

The preservation of life is the first priority at any major incident. The main aim of the disaster response teams is rescue and care of the survivors; thus, most of the disaster response plans do not cater the need of disaster victim identification issue [22]. The task of establishing the identity of victims of a mass disaster is termed as disaster victim identification (DVI) [23]. The identification is done primarily by parameters like visual acquaintance and fingerprints, but this use may be limited in case of mass disasters, burials, etc. where the human remains are destroyed beyond counts [24]. DVI plays an important role in disasters, especially in mass casualties associated with flood and earthquake disasters, the recurrence of these situations being very high in India and Nepal in the last few years. Following mass disaster, identification of individual victims by dental evidence is one of utmost reliable methods of identification in such situations [25]. Individuals with loss of all teeth can also be potentially identified based on the anatomy of the jaw bone or dentures with different shapes, sizes, manufacturers and compositions [26]. Moreover, dental data can also be used to determine individual's personal data such as age, race and gender [19]. Dental anomalies like missing teeth, supernumerary teeth and presence of extra cusp can also form important basis of identification of an individual [27].

The body identification guidelines are recommended by the American Board of Forensic Odontologists [28] for reporting dental identification. No doubt forensic dentistry has provided accurate identifications in mass disasters and 
other regular cases over the past years, but misidentifications have also occurred. These guidelines aim to provide guidance to the forensic dentist and are dedicated to the concept of greater accuracy in forensic dentistry.

\section{Collection and Preservation of Post-mortem Dental Evidence}

This is done under the authorization of a forensic pathologist. The regional coroner/medical examiner approves the decisions to incise the facial tissues for access or resect the jaws. The actual procedures to be followed in a dental identification case depend mostly on the condition of the remains.

\section{Sources for Ante-mortem Data}

Ante-mortem data obtained by investigative agency are dental radiographs, written records, etc. However, additional characteristics recognized by the forensic odontologists (e.g. prior orthodontic treatment) could be helpful in establishing a putative ID.

\section{Comparison of Ante-mortem and Post-mortem Evidence}

Factors such as teeth, missing teeth, tooth type, tooth position, crown morphology, crown pathology, root morphology, root pathology, pulp chamber and root canal morphology, pulp chamber and root canal pathology, dental restorations, periodontium, periodontal ligament, morphology/pathology, maxilla and mandible and other pathologic processes/jaw bones may be present in both the ante-mortem and post-mortem dental evidence and can be used for comparison. Most dental identifications are based on restorations, caries, missing teeth and/or prosthetic devices leading to easy documentation in the records.

\section{Categories and Terminology for Body Identification}

The categories and terminology used for body identification are described in Table 1.

Dental remains are fast, reliable and relatively inexpensive means of person identification during disasters. Scientific literatures have documented the role of dentists during various disaster-related events including earthquakes and terrorist attacks [29]. Dental professionals are compelled by law to produce and maintain adequate patient records, which provides valuable information about patients. The role of dentists has been reported in treating facial injuries sustained during the earthquake that struck China in 2008 [4]. About 8.9 per cent of the patients who sustained facial injuries in that earthquake were under the care and treatment of dental team members. Scientific documents [30] suggest that dental records indicate an increase in the number of illegal immigrants across international borders and a corresponding increase in the number of children without any valid proof of birth. This coupled with increasing natural disasters triggered by global climate change necessitates developing valid methods of estimation of age. A quick, valid and reliable method of age estimation during the times of disasters can be offered by dental tissues due to its resistance to both endocrinopathies and environmental insults.

\section{Dental Identification}

The dental identification (DI) highlights the role of teeth and is based on certain pathological conditions including prior dental treatment, malocclusion or disturbance in tooth eruption [19]. Tooth is the hardest structure of the body, which can withstand heavy forces and temperature. Therefore, the role of teeth or the dentition or dental treatments is preferable over other methods in the identification of the victims. As stated earlier, the dental tissue is resistant to trauma, mutilation, incineration and decomposition, thus making it an excellent reservoir of DNA material [31, 32].

\section{DNA in Dental Identification}

The failure of conventional dental identification methods increases the importance of biological material such as DNA to provide the necessary link to establish identity. The revolution in DNA technology has established the forensic DNA profiling as a gold standard for identification of unknown remains [23]. The objective of DNA profiling for disaster victim identification is to extract as much genetic information as possible from highly compromised samples [31].

Table 1 Categories and terminology for body identification

Positive identification

Possible identification

Insufficient evidence

Exclusion
Detailed matching of ante-mortem and post-mortem records with no unexplainable discrepancies

Ante-mortem and post-mortem data are consistent but due to poor quality identity cannot be positively established

Exact basis for a conclusion cannot be formed from available information

The ante-mortem and post-mortem data are clearly inconsistent 
Table 2 Catastrophe preparedness competencies

\begin{tabular}{ll}
\hline Competency & Description \\
\hline 1 & Adequate knowledge about the potential role of dentists in the first/early response in a range of catastrophic events \\
$\mathbf{2}$ & Appropriate description about the chain of command in the national, state and/or local response to a catastrophic event \\
$\mathbf{3}$ & Demonstration of the likely role of a dentist in an emergency response and participation in a simulation/drill \\
$\mathbf{4}$ & Demonstration of the possible role of a dentist at the level of a response team in all communications, the media, the general \\
public, and patient and family & Identification of personal limits as a potential responder and source that is available for referral \\
$\mathbf{6}$ & $\begin{array}{c}\text { Application of problem-solving and flexible thinking to unusual challenges within the dentist's functional ability and evaluation } \\
\text { of the effectiveness of actions that are taken }\end{array}$ \\
$\mathbf{7}$ & $\begin{array}{c}\text { Recognition of deviations from the norm, such as unusual cancellation patterns, symptoms of seasonal illnesses occurring } \\
\text { throughout the normal season, and employee absences, indicating an emergency and description of appropriate action }\end{array}$ \\
\hline
\end{tabular}

\section{Existing Curriculum in Dental Institutions}

As per the prescribed syllabus for Bachelor of Dental Surgery course by Dental Council of India as published in the gazette of India, all dental colleges impart subjective knowledge of anatomy (including general and dental), physiology, pathology (including systemic and oral), forensic odontology, microbiology, pharmacology, surgery and medicine (oral as well as general), public health, biostatistics and research methodology, practice management and ethics, thus making the dentist a potential first responder.

Moreover, hospital-based training and education (in medicine and surgery) are important components of specialized dental professional training including OMFS, general practice residency and many paediatric dentistry training [5], which is beyond that presented in a standard dental institution curriculum. Further, graduates of these hospital-based programs often continue to have hospital practices or affiliations. These competencies are based on the recognition that the knowledge and skills of an average dental graduate may be utilized by the public healthcare system in times of crisis [33]. However, a revised curriculum with the inclusion of handling of medical emergencies needs to be enforced in all dental teaching institutions.

\section{Recommended Expansion of the Curriculum}

The curriculum in dental schools should include training of students in a core set of competencies of mass disaster and bioterrorism (Table 2). A meeting was held by the American Dental Association (ADA) in June 2002 to identify the specific areas in which the dental profession could provide emergency assistance and prepare dentists adequately [34]. Dentists and their dental auxiliaries can augment the existing medical professionals in response to declared medical emergencies [35].
Apart from the above-mentioned competencies, an integrated fourth-year course in catastrophe preparedness can also be developed for dental students, as has been implemented in the dental curricula of New York University as a senior-level course [36]. The National Disaster Life Support (NDLS) training program can also be developed to better prepare healthcare professionals and emergency response personnel for mass casualty events [18]. This program consists of three levels of courses of increasing clinical complexity: (1) Core Disaster Life Support (CDLS), (2) Basic Disaster Life Support (BDLS) and (3) Advanced Disaster Life Support (ADLS) and can also be incorporated into the postgraduate training curriculum.

\section{Conclusion}

Dentists are well experienced in infection control procedures, taking and using information from medical histories to guide their actions, taking and interpreting radiographs, administering injections, suturing wounds, managing infections, prescribing medications and making diagnosis on the basis of clinical signs and symptoms. These skills are applicable directly to catastrophic response [37]. The established public health system and medical community members must understand that, in medical surge events, dental personnel form an additional source of assistance in response activities [11]. Current concerns regarding the use of biological warfares result from the increasing number of countries that are engaged in the widespread use of such weapons and their acquisition by terrorist groups. The need of the hour is to develop bio-defence by full international cooperation and to educate the likely target populations about precautions and protective measures to be taken in such attacks [38]. Dentists can provide a valuable service to their patients and communities by providing quality information regarding possibility of attacks, after effects of these attacks and appropriate steps to be taken in response to such attacks [39]. 


\section{Compliance with Ethical Standards}

Conflict of interest All the authors declare that there are no conflicts of interest to be disclosed.

Research Involving Human Participants and/or Animals This article does not contain any studies with human participants or animals performed by any of the authors.

Informed Consent For this type of study, format consent is not required.

\section{References}

1. Colvard MD, Lampiris LN, Cordell GA, James J, Guay A, Lee M et al (2006) The dental emergency responder, expanding the scope of dental practice. J Am Dent Assoc 137:468-473

2. Guay AH (2007) The role dentists can play in mass casualty and disaster events. Dent Clin N Am 51:767-778

3. Janssen JA, Lampiris LN (2007) Disaster response in Illinois: the role for dentists and dental hygienists. Dent Clin North Am 51:779-784

4. Wang L, Wei JH, He LS, Cao M, Cao J, Liu YP et al (2009) Dentist's role in treating facial injuries sustained in the 2008 earthquake in China, How dental professionals can contribute to emergency response. J Am Dent Assoc 140:543-549

5. Psoter WJ, Triola MM, Morse DE, Rekow EDE (2003) Enhancing medical and public health capabilities during times of crisis. NY State Dent J 69:25-27

6. Guay AH (2002) Dentistry's response to bioterrorism: a report of a consensus workshop. J Am Dent Assoc 133:1181-1187

7. Gallian JM (2004) Dentists can contribute expertise in a major public health disaster. J Calif Dent Assoc 32:701-708

8. Flores S, Mills SE, Shackelford L (2003) Dentistry and bioterrorism. Dent Clinc North Am 47:733-774

9. Lennquist $S$ (2003) Education and training in disaster medicine: time for a scientific approach. Int J Disaster Med 1:9-12

10. Bethesda MD (2011) National Institute of Dental and Craniofacial Research. Dentistry's role in responding to bioterrorism and other catastrophic events. http://www.nidcr.nih.gov/careersand training/dentistrycatastrophicevents.htm

11. Poster WJ, Park PJ, Boylan RJ, Morse DE, Glotzer DL (2008) National emergency response programs for dental health care professionals. J Am Dent Assoc 139:1067-1073

12. Tong DC (2008) The role of dentists in a mass casualty situation-a New Zealand perspective. J Mil Veterans Health $16: 20-25$

13. Giddon DB (2004) should dentists become "oral physicians"? Yes, dentists should become "oral physicians". J Am Dent Assoc 135(4):438-442

14. Morlang WM (1996) Dentistry's vital role in disaster preparedness. J Cali Dent Assoc 24:63-66

15. Psoter WJ, Herman NG, More FG, Park P, Robbins M, Rekow ED (2006) Proposed educational objectives for hospital based dentists during catastrophic events and disaster response. J Dent Edu 70:835-843

16. Nuzzolese E, Di Vella G (2007) Future projecting concerning mass disaster management: a forensic odontology prospectus. Int Dent J 57:261-266

17. Clark DH (1994) An analysis of the value of forensic odontology in ten mass disasters. Int Dent J 44:241-250

18. Coule PL, Horner JA (2007) National disaster life support programs, a platform for multi-disciplinary disaster response. Dent Clin N Am 51(4):819-825
19. Pretty IA, Sweet D (2001) A look at forensic dentistry-part 1: the role of teeth in the determination of human identity. Br Dent J 190:359-366

20. The Dental Industry and Japan Catastrophe (2011) Available from URL: http://worldental.org/dental-news/dental-industryjapancatastrophe/4289/

21. Dentists, Citizens Face 'Fearful Dark Clouds' After Earthquake, Tsunami (2011) Available from URL: http://www.ada.org/news/ 5639.aspx

22. Morris TB (2007) Managing the dead in catastrophes: guiding principles and practical recommendations for first responders. Int Rev Red Cross 89(886):421-442

23. Prinz M, Carracedo A, Mayr WR, Morling N, Parsons TJ, Sajantila A et al (2007) DNA commission of the international society for forensic genetics (ISFG): recommendations regarding the role of forensic genetics for disaster victim identification (DVI). Forensic Sci Int Genet 1(1):3-12

24. Petju AM, Suteerayongprasert RT, Hassiri K (2007) Importance of dental records for victim identification following the Indian Ocean tsunami disaster in Thailand. Public Health 121(4): 251-257

25. Martin-de las Heras VS, Marques T, Exposito N, Bohoyo JM (2000) The application of dental methods of identification to human burn victims in a mass disaster. Int $\mathrm{J}$ Legal Med 113:236-239

26. Eckert W (1997) Introduction to forensic sciences, 2nd edn. CRC Press, Boca Raton, FL

27. Malik P, Singh G, Gorea R, Jasuja OP (2012) Prevalence of developmental dental anomalies: a study of Punjabi population. Anil Aggrawal's Internet. J Forensic Med Toxicol 13(1):1-21

28. ABFO Reference Manual (2012) American board of forensic odontology. http://www.abfo.org/wp-content/uploads/2012/08/ ABFO-Reference-Manual-August-2012-revision.pdf

29. Hinchliffe JA (2007) Disaster dentistry. $\mathrm{Br}$ Dent J 202(8):493-494

30. Kieser JA, DeFeiter J, TeMoananui R (2008) Automated dental aging for child victims of disasters. Am J Disaster Med 3:109-112

31. Sweet D, Hildebrand D, Phillips D (1999) Identification of a skeleton using DNA from teeth and a PAP smear. J Forensic Sci 44:630-633

32. Budowle B, Bieber FR, Eisenberg AJ (2005) Forensic aspects of mass disasters: strategic considerations for DNA-based human identification. Leg Med 7:230-243

33. More FG, Phelan J, Boylan R, Glotzer DL, Psoter W, Robbins M et al (2004) Predoctoral dental school curriculum for catastrophe preparedness. J Dent Educ 68:851-858

34. Chmar JE, Ranney RR, Guay AH, Haden NK, Valachovic RW (2004) Incorporating bioterrorism training into dental education: report of the ADA-ADEA terrorism and mass casualty curriculum development workshop. J Dent Educ 68:1196-1199

35. Menon I, Hari Prakash G, Srivastava BK, Nagarajappa R, Tangde P (2010) "Bioterrorism"-knowledge, attitude and practice among faculty members of dental schools of Uttar Pradesh, India. J Ind Assoc Public Health Dent 15:141-148

36. Glotzer DL, More FG, Phelan J, Boylan R, Psoter W, Robbins M et al (2006) Introducing a senior course on catastrophe preparedness into the dental school curriculum. J Dent Educ 70(3):225-230

37. Rekow ED (2006) The dental team, a ready reserve or an overlooked resource? J Am Dent Assoc 137:432-434

38. Kumar A, Verma A, Yadav M, Sabri I, Asthana A (2011) Biological warfare, bioterrorism and bio-defence. J Indian Acad Forensic Med 33:60-73

39. Prakash N, Sharada P, Pradeep GL (2010) Bioterrorism: challenges and considerations. Forensic Dent Sci 2:59-62 